\title{
Hypersensitivity pneumonitis associated with the use of temozolomide
}

\author{
D. Koschel*, S. Handzhiev*, V. Leucht*, O. Holotiuk ${ }^{\#}$, \\ A. Fisseler-Eckhoff ${ }^{\star}$ and G. Höffken ${ }^{\star,+}$
}

ABSTRACT: The aim of the present study was to investigate a case of hypersensitivity pneumonitis associated with the use of temozolomide in the treatment of gliosarcoma.

A 54-yr-old female developed dyspnoea, cough and hypoxia after surgical resection for gliosarcoma and adjuvant radio- and chemotherapy with temozolomide. A high-resolution computed tomography scan of the thorax showed a bilateral ground-glass pattern. Bronchoscopy with bronchoalveolar lavage and lung biopsies was performed.

Bronchoalveolar lavage demonstrated significant lymphocytic alveolitis and transbronchial lung biopsies revealed lymphocytic infiltration with foamy macrophages, consistent with hypersensitivity pneumonitis. There was no evidence of other causes, including infections. After withdrawing temozolomide and initiating prednisolone therapy, the patient had no further pulmonary symptoms.

To the present authors' knowledge, this is the first definitively described case of temozolomideassociated hypersensitivity pneumonitis.

\section{KEYWORDS: Drug-induced lung disease, hypersensitivity pneumonitis, temozolomide}

$\mathbf{T}$ he new World Health Organization classification defines gliosarcoma as a glioblastoma variant characterised by a biphasic tissue pattern with alternating areas displaying glial and mesenchymal differentiation. Gliosarcoma is a relatively rare malignant neoplasm accounting for about $2 \%$ of all glioblastomas [1].

Temozolomide is an alkylating agent with antitumour and antimetastatic potential, which has been approved recently for the treatment of glioblastoma multiforme [2]. The current initial treatment of choice is surgical resection followed by radiotherapy and daily temozolomide. Mild respiratory symptoms have been described mainly when temozolomide is used in combination with other pulmonary toxic agents [3-5], however severe pulmonary toxicity is uncommon.

\section{CASE REPORT}

The patient was a 54-yr-old female, who had undergone stereotactic surgical resection of a gliosarcoma at the end of January 2007. Due to focal epileptic seizures, an anticonvulsant treatment with valproic acid was started and changed to levetiracetam after 2 weeks because of hepatic toxicity. In March 2007, hyperfractionated radiotherapy was initiated and, after a delay of 2 weeks, a low-dose $\left(75 \mathrm{mg} \cdot \mathrm{m}^{-2} \cdot\right.$ day $\left.^{-1}\right)$ of temozolomide was added. After simultaneous radioand chemotherapy, a reported slowly progressive shortness of breath with nonproductive cough was suggested to be a viral infection of the upper airways. In April 2007, the pre-existing dyspnoea deteriorated and the patient developed a fever with an elevated temperature of $39^{\circ} \mathrm{C}$. A chest radiograph showed patchy infiltrates in both lungs and a venous ultrasound revealed a thrombosis of the left poplitea vein. A highresolution computed tomography (HRCT) of the lung was performed which showed a diffuse ground-glass pattern in both lungs with slightly irregular intralobular septal thickening. The patient was transferred to the Fachkrankenhaus Coswig Centre for Respiratory Medicine (Coswig, Germany), for further management of suspected interstitial pneumonia.

On physical examination, the patient presented with tachypnoea and was afebrile. Blood gas analysis showed a respiratory failure with an oxygen tension of $6.0 \mathrm{kPa}$, carbon dioxide tension of $3.9 \mathrm{kPa}$ and $\mathrm{pH}$ of 7.46 . Auscultation of the lungs revealed inspiratory crackles in both lung
AFFILIATIONS

*Dept of Pulmonary Diseases, Fachkrankenhaus Coswig Centre for Pulmonary Diseases and Thoracic

Surgery, Coswig, and

\#Gemeinschaftspraxis für Pathologie, +Dept of Internal Medicine I,

University Hospital Carl Gustav Carus Dresden, Dresden, and

"Institute of Pathology and Cytology (IPZ), HSK Kliniken Wiesbaden, Wiesbaden, Germany.

CORRESPONDENCE

D. Koschel

Dept of Pulmonary Diseases,

Fachkrankenhaus Coswig

Centre for Pulmonary Diseases and

Thoracic Surgery

01640 Coswig

Germany

Fax: 49352365205

E-mail: dr.koschel@

fachkrankenhaus-coswig.de

Received:

January 112008

Accepted after revision:

October 162008

STATEMENT OF INTEREST

None declared.

European Respiratory Journal Print ISSN 0903-1936 Online ISSN 1399-3003 
bases. Laboratory tests revealed a D-dimer concentration of $2.66 \mathrm{mg} \cdot \mathrm{L}^{-1}$ (normal $<0.25 \mathrm{mg} \cdot \mathrm{L}^{-1}$ ), lactate dehydrogenase activity of $25.8 \mathrm{mmol} \cdot \mathrm{s}^{-1} \cdot \mathrm{L}^{-1}$ (normal $<2.5 \mathrm{mmol} \cdot \mathrm{s}^{-1} \cdot \mathrm{L}^{-1}$ ) and C-reactive protein concentration of $40 \mathrm{mg} \cdot \mathrm{L}^{-1}$ (normal $\left.<5 \mathrm{mg} \cdot \mathrm{L}^{-1}\right)$. Pulmonary function tests revealed moderate restriction (total lung capacity $2.63 \mathrm{~L}$ (58\% predicted), forced vital capacity $1.54 \mathrm{~L}$ (60\% pred), forced expiratory volume in one second $1.52 \mathrm{~L}(68 \%$ pred)) and a diffusion capacity of $1.23 \mathrm{mmol} \cdot \mathrm{min}^{-1} \cdot \mathrm{kPa}^{-1}$ (16\% pred). Echocardiography showed no pathologic findings. A chest radiograph (fig. 1a) showed a ground-glass pattern in both lungs with accentuation of the left lung, and an HRCT image of the lung with the patient prone (fig. 1b) demonstrated diffuse ground-glass opacities and the beginnings of a consolidation in the left lower lung. On contrast enhanced CT (fig. 1c) a pulmonary embolism of the left pulmonary artery was detected and therapy with low molecular weight heparin started. Bronchoscopy with BAL and transbronchial biopsies were performed. The BAL differential cell count showed $42 \%$ lymphocytes, $48 \%$ macrophages and 10\% neutrophils. Flow cytometric analysis of the BAL lymphocyte population revealed $97.5 \%$ of the lymphocytes were CD3+ Tcells with a CD4/CD8 ratio of 0.57 . In the fluid only Serratia spp. was detected. The biopsy specimens revealed a focal lymphocytic infiltration with free foamy intra-alveolar macrophages (mimicking a desquamative interstitial pneumonia-like pattern), a slight perivascular fibrin exudation and interstitial oedema consistent with a drug-induced hypersensitivity pneumonitis. They showed no evidence of granuloma, vasculitis or malignant infiltration (fig. $2 \mathrm{a}-\mathrm{c}$ ) and all cultures remained negative. A prednisolone therapy with $40 \mathrm{mg} \cdot \mathrm{day}^{-1}$ was initiated. Within 6 weeks of discharge, the patient's pulmonary symptoms resolved; on a follow-up visit in the clinic with current prednisolone $20 \mathrm{mg} \cdot \mathrm{day}^{-1}$, the blood gas analysis showed no more hypoxaemia, and a restrictive pulmonary function was no longer present. The diffuse ground-glass infiltrations had disappeared and only few residual signs remained (fig. 1d).
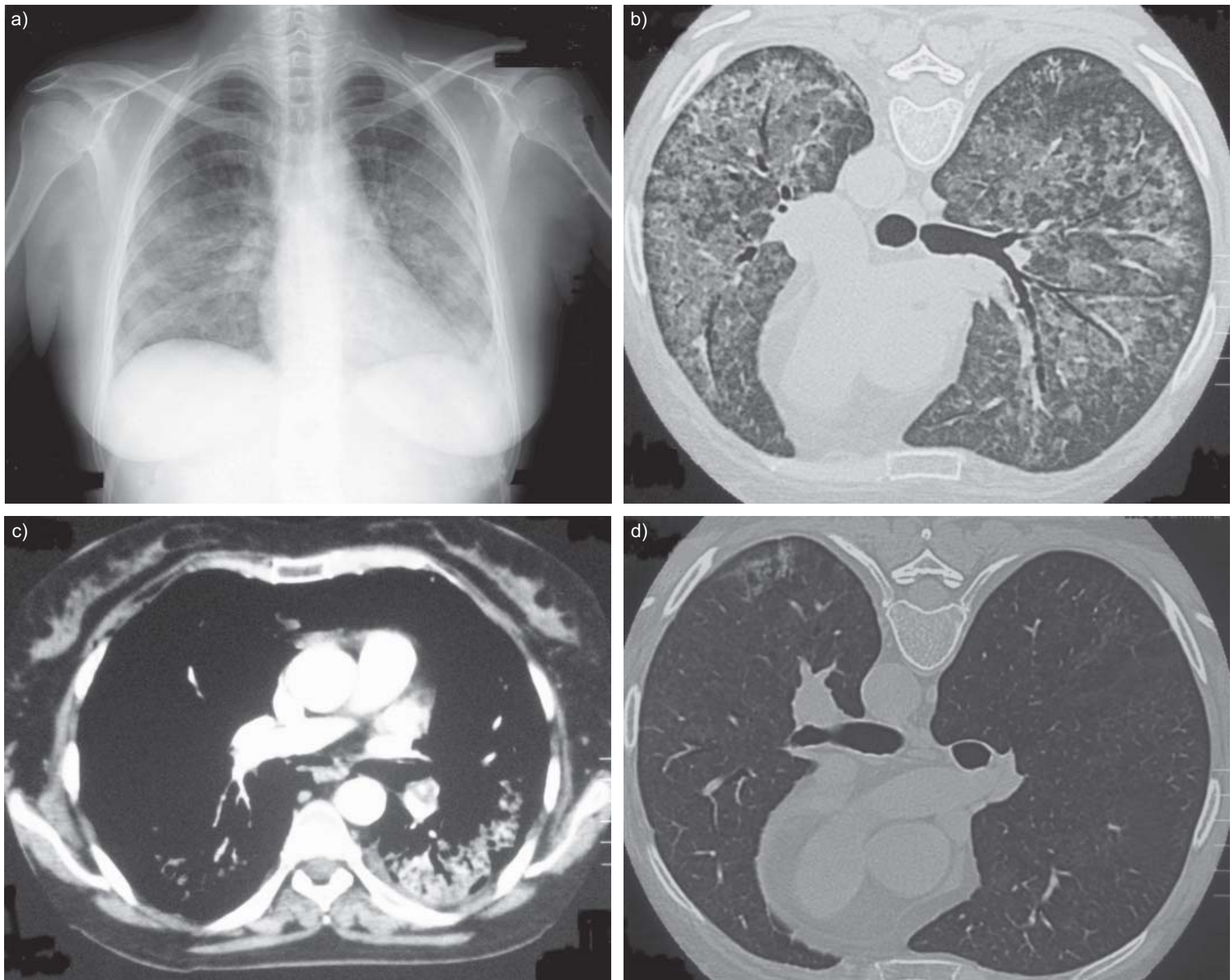

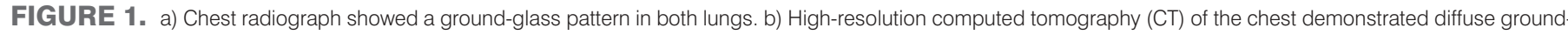

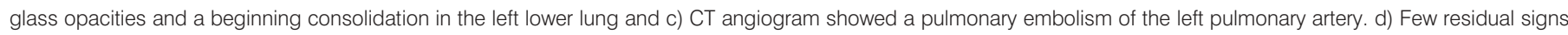
and no ground-glass opacities on a follow-up 6 weeks after discharge of the hospital and current prednisolone therapy. 

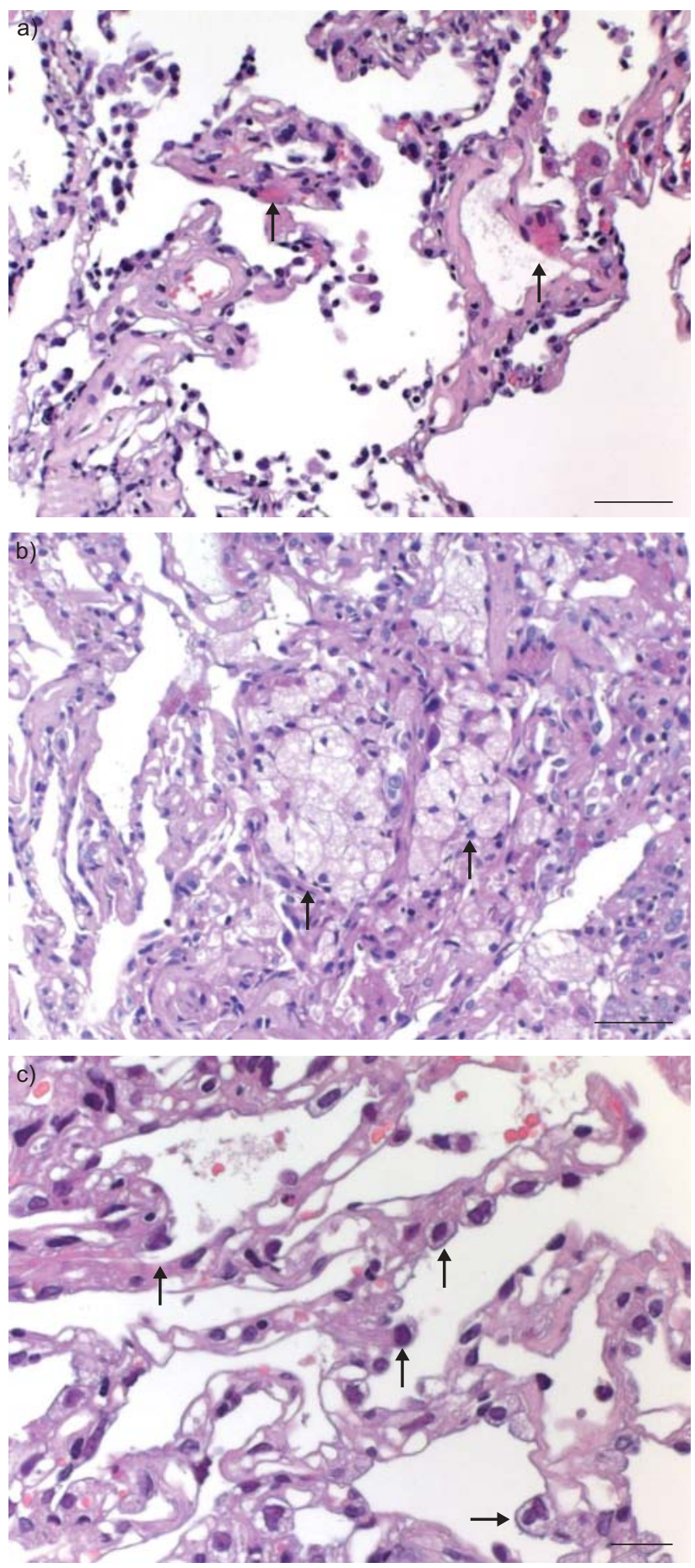

FIGURE 2. Transbronchial lung biopsies showed (arrows): a) a slight perivascular fibrin exsudation, b) free foamy intra-alveolar macrophages, which mimic a desquamative interstitial pneumonia-like pattern and c) proliferation of alveolar pneumocytes along alveolar septa. $a$ and b) Scale bars $=50 \mu \mathrm{m}$; c) Scale bar $=20 \mu \mathrm{m}$.

\section{DISCUSSION}

Many chemotherapeutic agents have been associated with pulmonary toxicities. Busulfan was the first chemotherapeutic drug with evidence of drug-induced lung disease [6]. Other alkylating agents, such as cyclophophamide and chlorambucil have been clearly associated with pulmonary toxicity as well [7].
Dacarbazine, which is closely related to temozolomide has been associated with pulmonary adverse effects only when used in combination with fotemustine, a nitrosourea agent [8].

Temozolomide is an imidazotetrazine compound and a derivative of the alkylating agent dacarbazine (second-generation oral alkylating agent). Temozolomide has proven activity against recurrent glioma [9]. In a recent randomised trial, concomitant and adjuvant temozolomide chemotherapy with radiation significantly improved progression free survival and overall survival in glioblastoma multiforme patients [2].

Various adverse reactions have been reported, but they are usually mild to moderate and in the majority of cases do not require a discontinuation of therapy. The haematological sideeffects are thrombocytopenia, neutropenia and particularly a CD4-selective lymphocytopenia with increased risk of opportunistic infections [10]. Because of the risk of Pneumocystis carinii pneumonia (PCP) infections, $\mathrm{PCP}$ prophylaxis is now recommended [11]. Other adverse effects of temozolomide such as alopecia, gastrointestinal, neurological, cardiovascular and neuromuscular have also been reported.

Respiratory toxicity has also been reported and the reports include dyspnoea, cough, upper respiratory tract infections, sinusitis and bronchitis. A high rate of thrombo-embolic events has been detected in patients treated with temozolomide in combination with thalidomide for metastatic melanoma [3] Pulmonary fibrosis has been reported in treatment regimes including agents with established pulmonary toxicity, such as carmustine or lomustine [4, 5]. In 41 patients with recurrent or progressive brain metastases undergoing temozolomide treatment $\left(150-200 \mathrm{mg} \cdot \mathrm{m}^{-2} \cdot \mathrm{day}^{-1}\right)$, two cases of mild interstitial pneumonitis were documented but no patient had to stop temozolomide due to toxicity [12]. Interstitial pneumopathy has been reported in patients with concomitant lympho- and/or granulocytopenia grade III-IV [13]. In a randomised controlled trial including $>500$ patients treated for glioblastoma with radiotherapy plus concomitant and adjuvant temozolomide, pneumonia was reported in three patients with radiotherapy plus temozolomide and in one with proven bacterial and candida pneumonia. No interstitial lung disease was observed [2]. In a study with temozolomide in paediatric solid tumours, pulmonary distress was seen in two cases: in one case as an interstitial pneumonia and in the other case as an asthma-like syndrome [14]. Recently, a case with temozolomide-associated lung disease has been published [15] but the histological examination of lung biopsy specimens was typical for organising pneumonia and had no signs of hypersensitivity pneumonitis.

Universally accepted criteria for the diagnosis of drug-induced pulmonary disease do not exist. Generally, it demands an appropriate history of drug exposure, histological evidence of lung injury and, most importantly, the exclusion of other causes of the lung damage [7]. In vitro tests are generally not recommended in drug-induced hypersensitivity pneumonitis $[7,16,17]$. Accordingly, these were not performed in the presented case. Reports of drug-induced lung disease with histological examination of lung tissue are $<10 \%$ of those published to date [16]. Drugs can induce many distinctive histopathological patterns of interstitial lung disease but cellular nonspecific interstitial pneumonitis is very common [16]. Free 
foamy intra-alveolar macrophages are also seen in druginduced lung disease, particularly in amiodarone pneumonitis [18]. They may be so numerous that they mimic the pattern of desquamative interstitial pneumonia [19]. Bronchoalveolar lavage in drug-induced pneumonitis may present any type of alveolitis (lymphocytic, neutrophilic, eosinophilic or mixed) or diffuse alveolar haemorrhage. The most frequent finding is a lymphocytic alveolitis with the predominance of CD8+ cells, just as in hypersensitivity pneumonitis [20].

In the present case, there are two competing issues: pulmonary embolism and the detection of Serratia spp. in bronchoalveolar fluid. Both issues could lead to the pulmonary status of the patient but it is unlikely that they were responsible for the diffuse ground-glass pattern in both lungs and the findings in BAL and lung specimens. The lymphocytic alveolitis in the BAL with low CD4/CD8 ratio and the lung biopsies revealing interstitial oedema, lymphocytic infiltration with intra-alveolar foamy macrophages particularly, were highly suggestive of hypersensitivity pneumonitis but not for pulmonary embolism or bacterial pneumonia.

Two other agents in the history of the patient must be checked. There are reports about pulmonary toxicity with valproic acid $[21,22]$ but none with interstitial lung disease. The patient had valproic acid for a few days only, since it had to be discontinued because of liver toxicity. The onset of pulmonary symptoms was not associated with concomitant drug administration. There are no reports on pulmonary toxicity of levetiracetam [23] and, therefore, therapy was maintained. It is unlikely that these two agents were responsible for the infiltrative lung disease.

The time course of the disease, the absence of alternative diagnosis, the radiological and bronchoscopy findings as well as the response to the treatment with prednisolone, strongly argue for hypersensitivity pneumonitis associated with the treatment with temozolomide. Rechallenging the patient with the putative agent to definitively verify the causal relationship is currently not recommended [7]. To the present authors' knowledge, this is the first case of temozolomide-associated hypersensitivity pneumonitis.

\section{REFERENCES}

1 Ohgaki H, Biernat W, Reis R, Hegi M, Kleihues P. Gliosarcoma. In: Kleihues P, Cavenee WK, eds. Pathology and Genetic of Tumors of the Nervous System. 2nd Edn. Lyon, IARC Press, 2000; pp. 42-44.

2 Stupp R, Mason WP, van den Bent MJ, et al. Radiotherapy plus concomitant and adjuvant temozolomide for glioblastoma. N Engl J Med 2005; 352: 987-996.

3 Krown SE, Niedzwiecki D, Hwu WJ, et al. Phase II study of temozolomide and thalidomide in patients with metastatic melanoma in the brain: high rate of thromboembolic events (CALGB 500102). Cancer 2006; 107: 1883-1890.

4 Chang SM, Seiferheld W, Curran W, et al. Phase I study pilot arms of radiotherapy and carmustine with temozolomide for anaplastic astrocytoma (Radiation Therapy Oncology Group 9813): implications for studies testing initial treatment of brain tumors. Int J Radiat Oncol Biol Phys 2004; 59: 1122-1126.
5 Herrlinger U, Rieger J, Koch D, et al. Phase II trial of lomustine plus temozolomide chemotherapy in addition to radiotherapy in neawly diagnosed glioblastoma: UKT-03. J Clin Oncol 2006; 24: 4412-4417.

6 Massin F, Fur A, Reybet-Degat O, Camus P, Jeannin L. [Busulfan-induced pneumopathy]. Rev Mal Respir 1987; 4: 3-10.

7 Limper AH. Chemotherapy-induced lung disease. Clin Chest Med 2004; 25: 53-64.

8 Gerard B, Aamdal S, Lee SM, et al. Activity and unexpected lung toxicity of the sequential administration of two alkylating agents - dacarbazine and fotemustine - in patients with melanoma. Eur J Cancer 1993; 29A: 711-719.

9 Yung WK, Albright RE, Olson J, et al. A phase II study of temozolomide versus procarbazine in patients with glioblastoma multiforme at first relapse. $\mathrm{Br}$ J Cancer 2000; 83: 588-593.

10 Brandes AA, Tosoni A, Cavallo G, et al. Temozolomide 3 weeks on and 1 week off as first-line therapy for reccurent glioblastoma: phase II study from groppo italiano cooperativo di neuro-oncologia (GICNO). Br J Cancer 2006; 95: 1155-1160.

$11 \mathrm{Su}$ YB, Sohn S, Krown SE, et al. Selective CD4+ lymphopenia in melanoma patients treated with temozolomide: a toxicity with therapeutic implications. J Clin Oncol 2004; 22: 610-616.

12 Abrey LE, Olson JD, Raizer JJ, et al. A phase II trial of temozolomide for patients with recurrent or progressive brain metastases. J Neuro Oncol 2001; 53: 259-263.

13 Chinot $\mathrm{O}$, Barrié $\mathrm{M}$, Cournède $\mathrm{A}$, et al. Phase II study of temozolomide (TMZ) administered on a 7 days on-7 days off regimen as primary treatment before radiotherapy (RT) in inoperable newly diagnosed glioblastoma multiforme (GBM). J Clin Oncol 2005; 23: Suppl. 16, 1523.

14 De Sio L, Milano GM, Castellano A, et al. Temozolomide in resistant or relapsed pediatric solid tumors. Pediatr Blood Cancer 2006; 47: 30-36.

15 Maldonado F, Limper AH, Lim KG, Aubrey MC. Temozolomide-associated organizing pneumonitis. Mayo Clin Proc 2007; 82: 771-773.

16 Camus P, Foucher P, Bonniaud P, Ask K. Drug-induced infiltrative lung disease. Eur Respir J 2001; 18: Suppl. 32, 93s-100s.

17 Sennekamp HJ. Drug-induced extrinsic allergic alveolitis. In: Sennekamp HJ, ed. Extrinsic Allergic Alveolitis/ Hypersensitivity Pneumonitis. 1st Edn. München/ Orlando, Dustri Verlag 2004; pp. 312-313.

18 Camus P, Martin WJ, Rosenow EC 3rd. Amiodarone pulmonary toxicity. Clin Chest Med 2004; 25: 65-75.

19 Morera J, Vidal R, Morell F, Ruiz J, Bernadó LL, Laporte JR. Pulmonary fibrosis and amiodarone. Br Med J (Clin Res Ed) 1982; 285: 895.

20 Costabel U, Uzalan E, Guzman J. Bronchoalveolar lavage in drug-induced lung disease. Clin Chest Med 2004; 25: 25-35.

21 Shaib FH, Patel B. A case of eosinophilic pleural effusion secondary to valproic acid. Chest 2000; 118: 308 S.

22 Sleiman C, Raffy O, Roue C, et al. Fatal pulmonary hemorrhage during high-dose valproate monotherapy. Chest 2000; 117: 613.

23 Pneumotox ${ }^{\circledR}$ Website, 1997. www.pneumotox.com Date last updated: August 2007. Date last accessed: April 2008. 